\title{
Comparison of different cutaneous carotenoid sensors and influence of age, skin type, and kinetic changes subsequent to intake of a vegetable extract
}

Martina C. Meinke

Sabine Schanzer

Silke B. Lohan

Ihar Shchatsinin

Maxim E. Darvin

Henning Vollert

Björn Magnussen

Wolfang Köcher

Jürgen Helfmann

Jürgen Lademann 


\title{
Comparison of different cutaneous carotenoid sensors and influence of age, skin type, and kinetic changes subsequent to intake of a vegetable extract
}

\author{
Martina C. Meinke, ${ }^{a, \star}$ Sabine Schanzer, ${ }^{a}$ Silke B. Lohan, ${ }^{a}$ Ihar Shchatsinin, ${ }^{b}$ Maxim E. Darvin, ${ }^{a}$ Henning Vollert, ${ }^{c}$ \\ Björn Magnussen, ${ }^{d}$ Wolfang Köcher, ${ }^{d}$ Jürgen Helfmann, ${ }^{b}$ and Jürgen Lademann ${ }^{a}$ \\ ${ }^{a}$ Charité - Universitätsmedizin Berlin, Department of Dermatology, Venerology and Allergology, Center of Experimental and \\ Applied Cutaneous Physiology, Charitéplatz 1, 10117 Berlin, Germany \\ bLaser- und Medizin Technologie GmbH Berlin, Fabeckstr. 60-62, 14195 Berlin, Germany \\ 'BioActive Food GmbH, Am Ihlsee 36a, 23795 Bad Segeberg, Germany \\ 'Opsolution GmbH, Wilhelmshöher Allee 273a, 34131 Kassel, Germany
}

\begin{abstract}
In the last decade, cutaneous carotenoid measurements have become increasingly popular, as carotenoids were found to be a biomarker of nutrition rich in fruits and vegetables, permitting monitoring of the influence of various stress factors. For such measurements, in addition to the specific and selective resonance Raman spectroscopy (RRS), newly developed low expensive small and mobile sensors that are based on spatially resolved reflectance spectroscopy (SRRS) are used for cutaneous carotenoid measurements. Human volunteers of different age exhibiting skin types I to III were investigated using RRS and two SRRS-based sensors to determine the influence of these parameters on the measuring results. In two studies on volunteers of either the same age or skin type, however, the respective other parameter being varied and no significant influences of age or skin type could be detected. Furthermore, the kinetic changes resulting from the intake and discontinued intake of a vegetable extract rich in carotenoids showed a good correlation among the three sensors and with the detected blood carotenoids. This illustrates that the SRRS-based sensors and RRS device provide reliable cutaneous carotenoid values independent of age and skin types I to III of the volunteers. ๑ 2016 Society of Photo-Optical Instrumentation Engineers (SPIE) [DOI: 10.1117/1.JBO.21.10.107002]
\end{abstract}

Keywords: reflectance spectroscopy; resonance Raman spectroscopy; curly kale extract.

Paper 160290R received May 9, 2016; accepted for publication Sep. 29, 2016; published online Oct. $20,2016$.

\section{Introduction}

It is generally accepted that nutrition rich in fruits and vegetables is beneficial for human health and wellbeing. ${ }^{1-4}$ This is due to the high amount of secondary plant components such as antioxidants. ${ }^{5}$ The majority of the essential antioxidants have to be taken up with food. Antioxidants protect the cells against oxidative stress, which could be induced by various factors, such as inter-alia, solar radiation, smoking, alcohol consumption, lack of sleep, and psychological stress. ${ }^{6-11}$ If the redox system of the body is out of balance, cell damage and cell death could occur leading to several pathological consequences, e.g., neurological, cardiovascular, or dermatological diseases. ${ }^{12-14}$

Often nutritional supplements are ingested to compensate for an insufficient intake of healthy food. However, average consumers are hardly able to assess the quality of commercially available supplements, and even for experts it is difficult to exactly determine the effects of these supplements. The problem is lack of clear and quickly available clinical endpoints. In the past, the skin carotenoids were found to be an easy-to-measure and reliable parameter to monitor the antioxidant status of the skin. ${ }^{15-17}$ Until recently, the skin carotenoids were determined using resonance Raman spectroscopy (RRS), which is a noninvasive, highly sensitive, and fast measuring method. ${ }^{18}$ The RRS results were correlated with several endpoints such as

*Address all correspondence to: Martina C. Meinke, E-mail: martina.meinke@ charite.de the total antioxidant status of the skin as determined by radical scavenging measurements, ${ }^{19,20}$ furrow and roughness measurements of the skin, ${ }^{21}$ and changes in collagen and elastin indices after irradiation. ${ }^{22}$ Furthermore, the skin carotenoids have shown to correlate with the blood carotenoids analyzed by highperformance liquid chromatography. ${ }^{23}$ In addition, the response to oxidative stress and to nutrition rich in carotenoids was extensively investigated. ${ }^{16,24,25}$ Even though the RRS systems are smaller and less expensive, new systems based on reflectance measurements are even smaller and cheaper which could improve the handling and increase the areas of application. ${ }^{26}$

Therefore, small, light-weight sensors were developed based on reflectance spectroscopy (RS) and calibrated with RRS. These small devices permit the detection of cutaneous carotenoids within a score from 0 for "no carotenoids" to 10 for "maximum concentration." ${ }^{27}$ First, studies using this miniaturized RS device were performed on healthy students and pregnant women to evaluate their antioxidant status depending on exogenous and endogenous parameters. ${ }^{28,29}$

In this study, the reliability of the cutaneous carotenoid sensors, which were recently developed based on spatially resolved reflectance spectroscopy (SRRS), was investigated for inter individual influences, such as skin type and age, which might affect the optical measurements. RRS measurements were performed for reference. For this purpose, subjects of different

$1083-3668 / 2016 / \$ 25.00$ (C) 2016 SPIE 
age and skin types I to III, according to the Fitzpatrick classification, ${ }^{30}$ were included in the studies. To explain the possible influences of skin type and to confirm the suitability of the measurements area, palm melanin and redness of these subjects were measured on the palm and the volar forearm, as the stratum corneum (SC) is less thick on the forearm and this area is less sun-protected. Furthermore, the response to an increase and decrease in skin carotenoids was evaluated during and after termination of the intake of a vegetable extract rich in carotenoids in comparison to a placebo (uptake study). For all the studies, two different SRRS sensors and the RRS system were applied to monitor the skin carotenoids noninvasively. Because blood carotenoids should not be influenced by skin type and age, blood samples were taken from all volunteers to monitor possible other influences (lifestyle, nutrition, and stress) on the subjects selected. The plasma from these samples was analyzed for its carotenoid concentration. The volunteers completed questionnaires on their nutritional habits and lifestyle patterns.

\section{Material and Methods}

\subsection{Subjects}

The influence of age was investigated on 33 healthy volunteers aged between 19 and 74 years with skin type II, according to the Fitzpatrick classification. ${ }^{30}$

For the investigation of the skin type dependency, 33 volunteers were recruited. The volunteers were aged between 25 and 35 years and of skin types I-III. For each skin type, 11 subjects were included.

To investigate the correlation between the different skin carotenoid sensors and kinetic changes due to the intake of a carotenoid-rich supplement, 30 healthy volunteers (13 male, 17 female, mean age 36.3 years, skin types I to III) were included in the study.

Excluded from these studies were with skin diseases, admission to an institution due to an administrative or judicial order (according to $\S 29 \mathrm{MPG}$ ), known drug addiction or alcoholism, and expectant or lactating women. In the uptake study, only subjects with skin carotenoid values below 6 were enrolled. The investigations were performed in accordance with the ethical guidelines of the Declaration of Helsinki and had been approved by the local Ethics Committee prior to starting the study. All volunteers gave their informed written consent.

\subsection{Resonance Raman Spectroscopy}

The cutaneous carotenoid concentration was determined by RRS. ${ }^{18}$ This noninvasive method was applied on the palm of the right hand, since carotenoid concentrations are particularly high in this area due to the thick SC. The carotenoids were resonantly excited using an argon laser operating at $488 \mathrm{~nm}$. The intensity of the strong carbon double-bond stretch vibration Raman line, measured at $1525 \mathrm{~cm}^{-1}$, was used for cutaneous carotenoid detection. Every measurement was conducted five times and the mean value was determined. The data are presented in arbitrary units giving values from 0 to 10 . Zero corresponds to values below the threshold and 10 is the highest value observed, corresponding to $\sim 1 \mathrm{nmol}$ carotenoids/g skin. ${ }^{18}$

\subsection{CaroLED Carotenoid Concentration Sensor}

The CaroLED sensor (Laser- und Medizin-Technologie Berlin, LMTB, Germany, Fig. 1) is intended to quantify the carotenoid

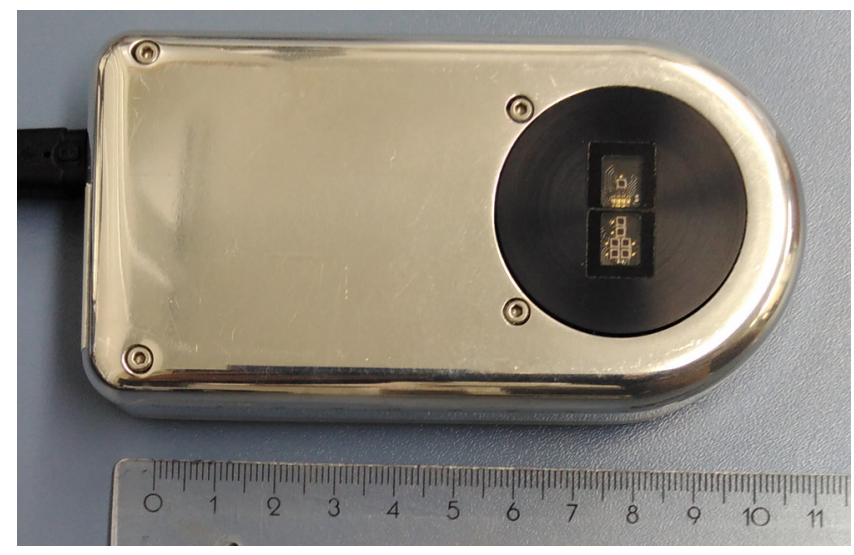

Fig. 1 Image of CaroLED sensor.

concentration in human skin. It was specifically calibrated for measurements on the palm of the hand. In previous measurements with RRS and CaroLED on the same subjects, a calibration data set was acquired. After preprocessing of the raw CaroLED data, a multivariate calibration based on partial least squares regression was performed. This calibration with RRS as the reference method gives carotenoid levels on a relative scale. The data acquired in the study presented in this publication were not part of the calibration procedure.

The underlying measuring principle of CaroLED is based on SRRS. ${ }^{31-34}$ Five light-emitting diodes (LED) of different wavelengths in the visible and near-infrared region are illuminating the skin through a measurement window. While light is propagating through the individual skin layers, the spectral signature of carotenoids and other skin constituents is obtained. ${ }^{35}$ Four photodiodes, each of which is placed at a different lateral distance to the illumination site, measure the spectral signature at different depth sensitivities. Combining this information, the influence of skin variance regarding light scattering and disturbing chromophores such as hemoglobin or melanin on the carotenoid measurement is minimized. The measurements were performed five times on the palm of the right hand.

\subsection{Multiple Spatially Resolved Reflection Spectroscopy Sensor}

The multiple spatially resolved reflection spectroscopy (MSRRS) sensor (Opsolution, Kassel, Germany) is an optical sensor based on MSRRS which is commercially available under the trade name biozoom (model Biozoom Portable). The MSRRS sensor uses several differently positioned light sources and light detectors (Fig. 2), exhibiting different distances between light source

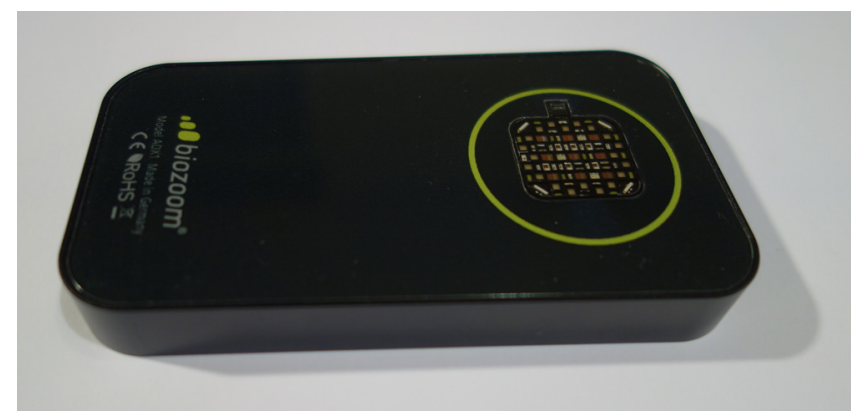

Fig. 2 Photograph of the MSRRS sensor. 
and detector and different angles for irradiation and detection. The measurement time for one measurement takes $\simeq 15 \mathrm{~s}$.

With the MSRRS sensor, it is possible to get reliable information, even if the sample is inhomogeneous or shows a gradient in substance concentrations. This makes the MSRRS sensor less sensitive to errors caused by the position of the measurement, as it averages the properties of the sample over a larger area.

The LED light sources cover a spectral range from $\sim 350$ to $1000 \mathrm{~nm}$ wavelength in 16 steps providing 118 light emitters. The sensor picks up the backscattered light at a total number of 152 light-sensitive areas. This combination results in almost 18,000 raw data values that are picked up several times during one measurement at a sensor surface area of $20 \mathrm{~mm} \times 20 \mathrm{~mm}$. The sensor was calibrated to the RRS using more than 3000 measurements. ${ }^{36}$ The calibration was computed by using a genetic algorithm on data pairs of preprocessed MSRRS raw data and RRS data to determine structure and parameters of the evaluation algorithm. Since the MSRR sensor is finally intended for use under everyday life conditions, the MSRRS data were collected in a previous calibration study on different volunteers, whereby the ambient temperatures and surface contact parameters were artificially varied. Therefore, the measurements in the present study are the blind test for the MSRRS sensor. The measurements in this study were performed five times on the palm of the right hand.

\subsection{Blood Carotenoids}

For determining the total blood carotenoids, ethylenediaminetetraacetic acid (EDTA) blood was sampled and centrifuged to separate the cells from plasma. An amount of 400- $\mu$ 1 EDTA plasma was taken to analyze the blood carotenoid content using a mobile device iCheck ${ }^{\mathrm{TM}}$ (BioAnalyt, Germany).

\subsection{Skin Parameters Melanin and Redness}

The skin parameters such as melanin and redness were measured using the Mexameter ${ }^{\circledR}$ MX 18: (Courage + Khazaka electronic $\mathrm{GmbH}$, Cologne, Germany). The device measures melanin and hemoglobin (erythema or redness) based on RS.

\subsection{Study Protocols}

For the age- and skin-type studies, the volunteers were measured at every visit with all three skin sensors and blood was sampled afterward. Furthermore, a questionnaire was completed on lifestyle, nutrition, and stress conditions. Before the measurements started, the subjects were asked to acclimate for 5 to $10 \mathrm{~min}$. In addition, they had been instructed not to use any skin care products $24 \mathrm{~h}$ before investigation.

For the uptake study, the volunteers were investigated at five visits. At every visit, the volunteers were measured five times with each of the three skin carotenoid sensors. Data analysis was based on the mean values of the repeated measurements. At the first visit, exclusively volunteers exhibiting values below 6 were screened using the RSS device as specifically subjects with a low to medium skin carotenoid status were in the focus of these investigations. Subsequently, the same volunteers were measured with all three cutaneous carotenoid sensors on the right palm. Afterward, their blood was sampled to determine the blood carotenoid concentrations and they completed the questionnaire. The same procedure was performed after 6 ,
12 , and 14 weeks of intake as well as 3 weeks after discontinuance of the supplementation.

\subsection{Vegetable Extract}

During the uptake study, the volunteers ingested three capsules of a curly kale/red pepper extract once a day (provided by BioActive Food, Bad Segeberg, Germany), hereinafter called verum. One capsule contained the following substances: $400 \mu \mathrm{g}$ lutein, $70 \mu \mathrm{g}$ beta-carotene, $30 \mu \mathrm{g}$ lycopene, and $20 \mu \mathrm{g}$ zeaxanthin. As a control, a placebo capsule containing no antioxidants was given.

\subsection{Questionnaire}

In the study, a questionnaire was prepared and subsequently completed by the volunteers to monitor their nutritional habits and lifestyle patterns, such as alcohol consumption, smoking, sun exposure, and stress. ${ }^{37}$

Dietary intakes of fruits and vegetables were examined by self-assessment based on a food frequency questionnaire with two separate food lists containing 13 common fruits and 13 common vegetables that had to be marked in a frequency category ("rarely," " $\geq$ once a month," " $\geq$ once a week," and " $\geq 4$ times a week").

\subsection{Statistical Analysis}

A descriptive explorative data analysis was performed using SPSS statistics $\left(\right.$ IBM $^{\circledR}$ SPSS $^{\circledR}$ Statistics, version 19, Inc. Chicago, Illinois). Since the obtained data were not normally distributed, nonparametric tests were performed. For independent data, the Mann-Whitney U test was applied, and for related data, the Wilcoxon test was applied. Values of $p \leq 0.05$ were considered to indicate a difference. To evaluate the significance over the entire measurement period, generalized estimating equation was performed; a $p$ value $\leq 0.05$ was considered as significant.

Correlation data were determined using Excel by calculating the Pearson correlation coefficients.

\section{Results}

\subsection{Influence of Age}

To investigate the influence of the age, 33 volunteers of skin type II aged from 19 to 79 years, were investigated with all three skin sensors, whereas blood samples were taken to analyze the total amount of blood carotenoids. Three age groups of 11 subjects, each, were differentiated: group one consisting of volunteers below 30 years, group two comprised volunteers aged between 31 and 55 years, and the volunteers of the last group were older than 55 years. The mean values and standard deviations determined per group and detection method are shown in Fig. 3. No significant differences in skin or blood carotenoids were found among the three age groups.

\subsection{Influence of Skin Types I to III}

The influence of the skin type was investigated by including 33 volunteers of skin type I $(n=11)$, skin type II $(n=11)$, and skin type III $(n=11)$, respectively. The volunteers were aged $30 \pm 5$ years. Blood was measured as a reference because the blood values should not be influenced by the skin type. The 


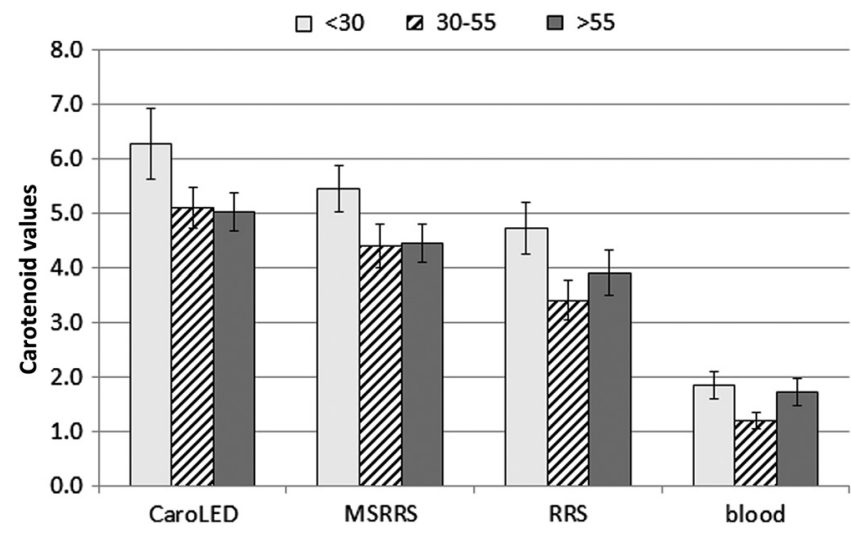

Fig. 3 Mean values and SEM of skin and blood carotenoids for three different age groups, 19 to 30 years, 30 to 55 years, and 55 to 79 years, $n=11$ for each group, skin type II. Values for skin carotenoids are shown in a.u. for blood carotenoids in $\mathrm{mg} / \mathrm{L}$.

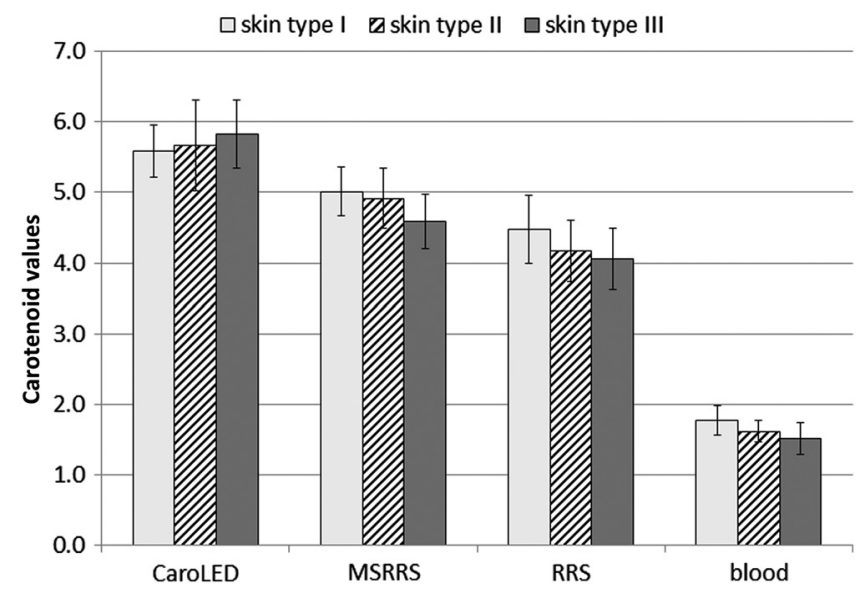

Fig. 4 Skin and blood carotenoids (mean \pm SEM) for skin types I, II, and III and mean age $30 \pm 5$. Skin carotenoids are shown in a.u., blood carotenoids in $\mathrm{mg} / \mathrm{L}, n=11$ for each skin type.

mean values and standard deviations for the skin and blood carotenoids per group and detection method are shown in Fig. 4.

No statistically significant differences in skin and blood carotenoids were found for the different skin types.

Furthermore, the melanin content and the redness of the skin were measured for selected volunteers (Fig. 5).

The melanin content of the palm increased with increasing skin type but this is more pronounced on the inner forearm. The redness at the palm shows higher values than on the arm. At the forearm, a significant difference between skin types I and II could be observed for redness $(p=0.003)$, while for melanin a trend was observed $(p=0.072)$. Regarding differences between skin types II and III, again only at the forearm the values showed a trend for melanin $(p=0.052)$. However, for all data in Fig. 5, significant differences between skin types I and III appeared $(p<0.05)$ with the exception of redness at the palm; here only a trend was visible $(p=0.051)$.

\subsection{Kinetics of Cutaneous Carotenoids}

The uptake study was performed on 30 volunteers, 13 male, 17 female, skin types I, II, and III, mean aged 36.3 years ( 15 verum and 15 placebo) from October 2014 to March 2015. After visit 1,

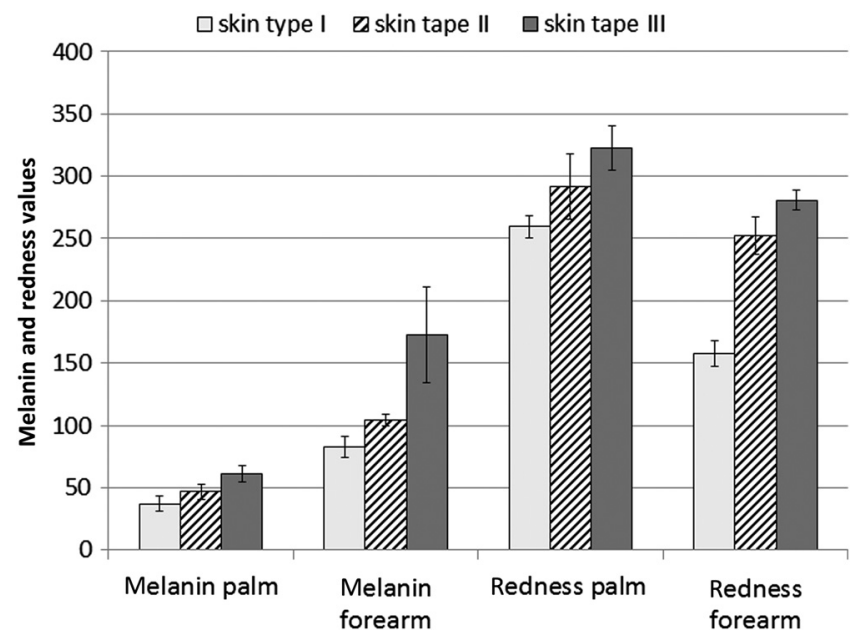

Fig. 5 Melanin and redness values (mean \pm SEM) on palm and inner forearm of the volunteers of different skin types. Number of volunteers, skin type $\mathrm{I}=7$, skin type $\mathrm{II}=5$, and skin type $\mathrm{III}=6$.

the volunteers started to ingest the vegetable extract or the placebo sample for 14 weeks. The skin carotenoids were measured with all three skin sensors on the palm of the right hand, the blood carotenoids were determined and the questionnaire was completed before, 6,12 , and 14 weeks after supplementation and 3 weeks after the discontinuance of supplementation to see if either skin or blood carotenoids or both had decayed.

To demonstrate the kinetics of the values, the blood and skin carotenoid concentrations relative to the initial values are shown with their mean and standard deviations in Figs. 6 and 7.

The blood values increased already after 6 weeks of intake to the maximum level (55\% increase) and remained almost stable until the intake was discontinued. After stopping the intake, the values decreased almost to the initial value. The placebo values also increased by $20 \%$ and decreased after discontinuing the intake.

In contrast to the blood values, the skin values still increased for a period between 6 and 12 weeks and remained almost stable for the next 2 weeks of intake. This study illustrated for the first time that the regular intake of a moderate dose of antioxidants leads to stable skin carotenoid values. When the intake was discontinued, the values decreased although at a smaller rate compared to the blood values. The maximum increase of the skin carotenoids was dependent on the method used. With 48\%, RRS exhibits higher changes. Both reflectance measurements reached

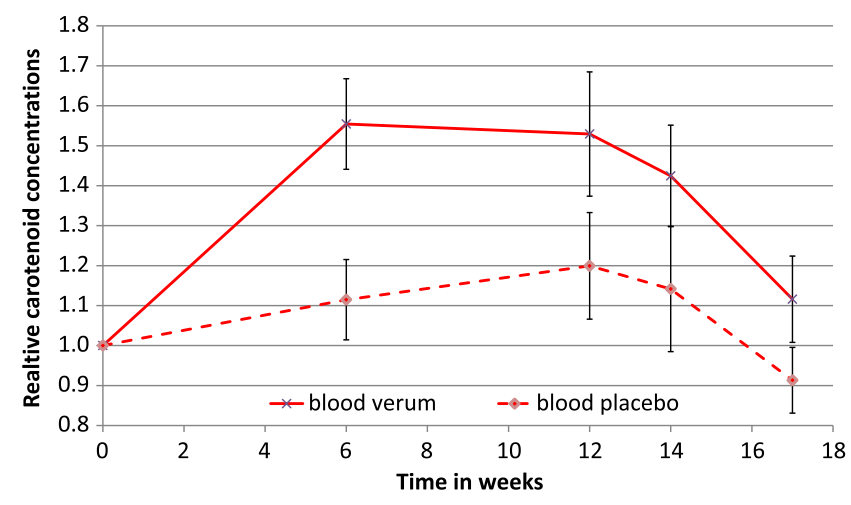

Fig. 6 Mean and SEM of blood carotenoids relative to initial values of individual measurements. 


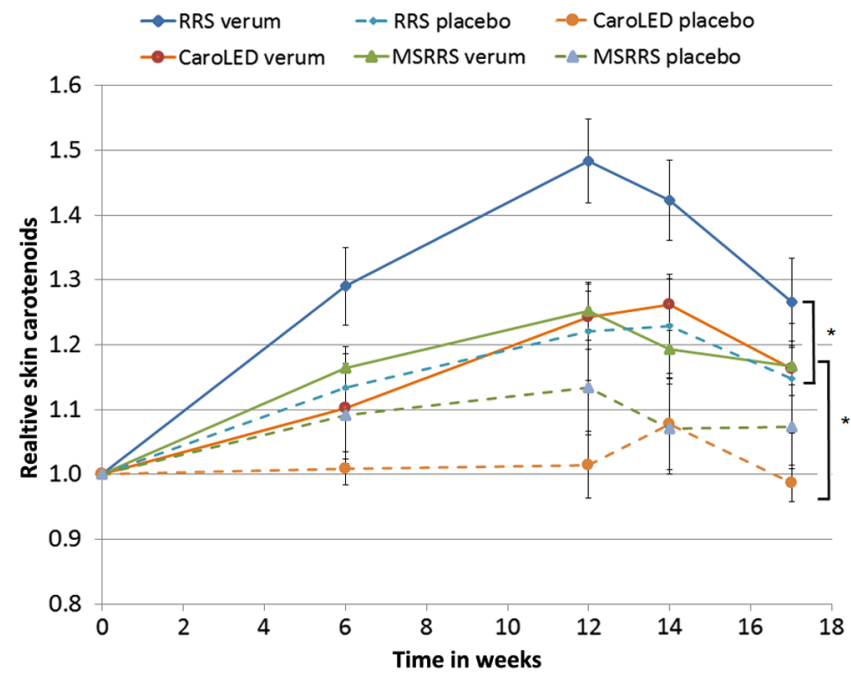

Fig. 7 Mean and SEM of skin carotenoids relative to initial values of the placebo and verum groups measured by the two SRRS and the RRS systems.

$\sim 26 \%$ increase. As observed in blood, an increase in cutaneous carotenoid values was also obtained in the placebo group.

To investigate the significance of difference between placebo and verum, the Mann-Whitney $U$ test was applied for all time points and sensors. The $p$ values are given in Table 1 .

The questionnaires were analyzed for changes in nutrition or stress factors over the duration of the study. Significant changes could be found neither in the verum nor in the placebo group.

\subsection{Correlations Between the Skin and Blood Carotenoid Values}

To validate the sensors, the correlation of the carotenoid values between the two reflectance-spectroscopy-based skin sensors and the RRS device, and the correlation to the blood values of all three noninvasive sensors were calculated using Excel (Table 2). As can be seen in Fig. 7, the absolute values given by the sensors are not identical. The correlation between the sensors was used for comparison purposes to neutralize effects caused by the sensors scaling the displayed output values differently. The MSRRS and CaroLed sensors are scaling the output so that an increase of the carotenoid levels results in a smaller increase of the displayed values compared to the RRS measurement. There is also an offset among the different sensors. The different scaling also explains the different absolute values seen in Fig. 6. Offsets and constant scaling factors are eliminated by using correlation values providing an objective comparison.

Table 1 Calculated $p$ values for all time points and sensors between placebo and verum using the Mann-Whitney $U$ test; in brackets, the significance level is clarified.

\begin{tabular}{lcccc} 
& V2 & V3 & V4 & V5 \\
\hline CaroLED & 0.06 & $0.001\left(^{* \star}\right)$ & $0.019\left(^{\star}\right)$ & $0.002\left(^{* \star}\right)$ \\
MSRRS & 0.061 & $0.03\left(^{*}\right)$ & $0.023\left(^{*}\right)$ & $0.045\left(^{*}\right)$ \\
RRS & 0.098 & $0.015\left(^{*}\right)$ & $0.005\left(^{* \star}\right)$ & 0.089 \\
Blood & $0.011\left(^{*}\right)$ & $0.026\left(^{*}\right)$ & $0.029\left(^{*}\right)$ & 0.174 \\
\hline
\end{tabular}

Table 2 Pearson correlation coefficient $R$ for the data of the uptake study $(n=150)$.

\begin{tabular}{lccc} 
& CaroLED & MSRRS & RRS \\
\hline RRS & 0.7433 & 0.7895 & \\
Blood & 0.6215 & 0.6672 & 0.6540 \\
\hline
\end{tabular}

The comparison of the carotenoid values obtained using reflectance-based sensors and the RRS system demonstrate a good correlation. The MSRRS data from the first visit show significantly lower correlation than visits two to five, leading to the assumption that in this series an error occurred during the data collection. For the MSRRS and the CaroLED sensor, the study was a valid blind test, as the data of the study were not used for the calibration of the algorithms.

Based on the uptake study, the reproducibility of measurements conducted with the three carotenoid sensors was compared by calculating the mean standard deviation of five measurements performed at every visit and are shown in Table 3.

The results show that the reproducibility of RRS is slightly below that of the MSRRS and CaroLED sensors.

\section{Discussion}

The investigation using different sensors showed that the influence of age on the skin carotenoid concentration is not significant. This data correlate with the former study on 150 subjects over a broad age range using RRS. ${ }^{38}$ In this former study also no significant changes in the total carotenoid concentration were detected. Only in older male subjects, the lycopene decreased significantly, which was not in the focus of the present study.

The skin carotenoid values of all three sensors correlate with the blood values. There are no significant differences in correlation to blood values between the RS-based sensors and the RRS system. The correlations are in agreement with other studies using RRS. ${ }^{23,39-42}$ At steady state situation, the skin carotenoids reflect the blood values. Only if fast strong changes occur, as observed in the uptake study, the blood values increase and decrease faster compared to the cutaneous values (Fig. 6 versus Fig. 7).

This suggests that an accumulating effect in the skin might delay the response of the cutaneous values to occurring changes. As the SC in the palmar region is thicker than on other sites of the body, the palm of the hand was chosen for the measurements. The SC acts as a reservoir for carotenoids, mostly providing a stronger signal than other skin areas. ${ }^{18}$

No effects of skin types I, II, and III could be found by measuring carotenoids on the palm using the three sensors. This could be due to the little changes in melanin on the palm (Fig. 5) even if they were significant. ${ }^{43} \mathrm{~A}$ small variance in

Table 3 Calculated mean standard deviations of all five repetition skin carotenoid measurements for the applied devices during the uptake study.

\begin{tabular}{lccc} 
& CaroLED & MSRRS & RRS \\
\hline Visits 1 to 5 & 0.22 & 0.23 & 0.32 \\
Visits 2 to 5 & 0.21 & 0.22 & 0.31 \\
\hline
\end{tabular}


redness had no significant influence on the carotenoid values in the palm. Compared to other skin sites, the 120 - to $150-\mu$ m-thick $\mathrm{SC}$ on the palm reduces the influence of blood, which could reduce the signal due to the high absorption of light in the excitation wavelength used. ${ }^{44}$ This illustrates that the measurement area at the palm was a good choice for minimizing possible influences. Nevertheless, we cannot exclude influences in the case of skin types higher than III. Using a Konica Minolta CM2600d reflectance spectrophotometer, Coetzee and Perrett ${ }^{45}$ investigated dermal changes in African volunteers induced by beta-carotene supplementation. They found that changes occur at the palm and the inner forearm but not on sun-exposed areas. A study comparing skin carotenoids by RS in AsianKorean, German-Korean, and Caucasian skin illustrated that the changes in skin carotenoids were related to nutrition and stress, whereas an influence of the skin type was not reported. ${ }^{46}$

Regarding the kinetic changes, again the differences between blood and cutaneous carotenoids appeared as shown earlier by Meinke et al. ${ }^{23}$ Interestingly, when the skin carotenoids reach a plateau during supplementation, the RRS values reach almost the same increase (by a factor of 1.5) as the blood values. This enhancement is quite high; in former studies over a shorter time period smaller effects were observed. ${ }^{23,47}$ This could be due to the fact that in these studies no saturation was achieved.

The skin carotenoid values obtained using the reflectancebased sensors did not increase as much as the RRS values. The observed differences are caused by the sensors using a different scaling of the output. Having a more detailed look at the data, it is noticeable that the correlation factor between MSRRS sensor and RRS for the first visit was nearly $50 \%$ less compared to all other visits. The correlation of visits 2 to 5 varied only between $2 \%$ and $10 \%$. Applying the same device on the same volunteers with such a difference suggests that the data collection for visit one was associated with an incomplete covering of the sensor area. After visit 1, the sensor was improved with a software that detects such failures and incorrect measurements could be repeated. Excluding the data of visit 1 from the calculation of the correlation factor the value increased (0.8363).

The correlation among the three sensors showed that the calibration to the RRS was successful but could be improved. However, it had to be taken into account that the correlation was restricted by the RRS standard deviation. In this study, therefore, the RRS device was modified to integrate the backscattering signal in the analysis. Although the device was adjusted to the former condition by phantoms, an influence on the correlation between the RS and Raman spectroscopybased sensors cannot be excluded.

A significant increase in cutaneous and blood carotenoids was observed for the placebo group in the uptake study. This increase could not be explained by changes in the nutritional or lifestyle habits, as the analysis of the questionnaire did not reveal significant changes. However, as the increase became obvious after the Christmas holidays, a reduction in stress cannot be excluded. Furthermore, curly kale is a traditional German Christmas and winter vegetable and the subjective questionnaires did not reflect all changes because they were not completed daily but in a summarized manner at every visit.

\section{Conclusion}

The three studies have shown that the carotenoid measuring values of all sensors are independent of age and skin types (I to III), and all three systems are capable of monitoring carotenoid kinetic changes, which result from changes in nutrition. As a result, the palm as the measurement area was confirmed to be well suitable because of its low pigmentation and a thick SC. Compared to the RRS system, the reflectance-spectroscopy-based sensors provided a slightly reduced sensitivity, whereas the RRS standard deviation was higher than that of the two RS-based sensors. Nevertheless, the correlation among the sensors was around 0.7 and all skin carotenoids correlated with the blood carotenoid values. This illustrated that the RSbased sensors provide reliable results and are therefore suitable for monitoring changes, although with slightly less amplitude compared to the RRS system.

\section{Acknowledgments}

The study was supported by the German Federal Ministry of Education and Research (BMBF), funding code 13N12593. Bjorn Magnussen is the chief technology officer and Wolfgang Kocher is on the scientific board of biozoom Services GmbH, Kassel (Germany). Jürgen Helfmann is the research director of biomedical optics of the nonprofit research organization, Laserund Medizin-Technologie (LMTB) GmbH, Berlin (Germany). $\mathrm{H}$. Vollert is the head of BioActive Food GmbH, Bad Segeberg (Germany) providing the supplement. Jürgen Lademann is on the scientific board of biozoom services $\mathrm{GmbH}$, Kassel (Germany).

\section{References}

1. M. Fito and V. Konstantinidou, "Nutritional genomics and the mediterranean diet's effects on human cardiovascular health," Nutrients $\mathbf{8}(4)$, 218 (2016).

2. S. Li et al., "Healthful dietary patterns and the risk of hypertension among women with a history of gestational diabetes mellitus: a prospective cohort study," Hypertension 67(6), 1157-1165 (2016).

3. Y. Sun et al., "A healthy dietary pattern reduces lung cancer risk: a systematic review and meta-analysis," Nutrients 8(3), 134 (2016).

4. I. H. Dias et al., "Plasma levels of HDL and carotenoids are lower in dementia patients with vascular comorbidities," J. Alzheimers Dis. 40(2), 399-408 (2014).

5. M. C. Polidori et al., "Conflict of evidence: carotenoids and other micronutrients in the prevention and treatment of cognitive impairment," BioFactors 38(2), 167-171 (2012).

6. M. Khoubnasabjafari, K. Ansarin, and A. Jouyban, "Reliability of malondialdehyde as a biomarker of oxidative stress in psychological disorders," Bioimpacts 5(3), 123-127 (2015).

7. U. Mons et al., "Effect of smoking reduction and cessation on the plasma levels of the oxidative stress biomarker glutathione-post-hoc analysis of data from a smoking cessation trial," Free Radical Biol. Med. 91, 172-177 (2016).

8. E. Birben et al., "Oxidative stress and antioxidant defense," World Allergy Organ J. 5(1), 9-19 (2012).

9. A. Yoshida et al., "Blue light irradiation-induced oxidative stress in vivo via ROS generation in rat gingival tissue," J. Photochem. Photobiol. B 151, 48-53 (2015).

10. S. Grether-Beck et al., "French maritime pine bark extract (Pycnogenol (r)) effects on human skin: clinical and molecular evidence," Skin Pharmacol. Physiol. 29(1), 13-17 (2016).

11. C. M. Schempp et al., "Effect of topical application of Hypericum perforatum extract (St. John's wort) on skin sensitivity to solar simulated radiation," Photodermatol. Photoimmunol. Photomed. 16(3), 125-128 (2000).

12. D. Moretti et al., "Calpain-3 impairs cell proliferation and stimulates oxidative stress-mediated cell death in melanoma cells," PLoS One 10(2), e0117258 (2015).

13. Z. V. Varga et al., "Interplay of oxidative, nitrosative/nitrative stress, inflammation, cell death and autophagy in diabetic cardiomyopathy," Biochim. Biophys. Acta-Mol. Basis Dis. 1852(2), 232-242 (2015). 
14. Y. J. Xin et al., "Tet1-mediated DNA demethylation regulates neuronal cell death induced by oxidative stress," Sci. Rep. 5, 7645 (2015).

15. J. Lademann et al., "Cutaneous carotenoids: the mirror of lifestyle?" Skin Pharmacol. Physiol. 27(4), 201-207 (2014).

16. S. T. Mayne et al., "Noninvasive assessment of dermal carotenoids as a biomarker of fruit and vegetable intake," Am. J. Clin. Nutr. 92(4), 794-800 (2010).

17. S. T. Mayne et al., "Resonance Raman spectroscopic evaluation of skin carotenoids as a biomarker of carotenoid status for human studies," Arch. Biochem. Biophys. 539(2), 163-170 (2013).

18. M. E. Darvin et al., "Non-invasive in vivo determination of the carotenoids beta-carotene and lycopene concentrations in the human skin using the Raman spectroscopic method," J. Phys. D: Appl. Phys. 38(15), 2696-2700 (2005).

19. S. F. Haag et al., "Determination of the antioxidative capacity of the skin in vivo using resonance Raman and electron paramagnetic resonance spectroscopy," Exp. Dermatol. 20(6), 483-487 (2011).

20. S. F. Haag et al., "Comparative study of carotenoids, catalase and radical formation in human and animal skin," Skin Pharmacol. Physiol. 23(6), 306-312 (2010)

21. M. Darvin et al., "Cutaneous concentration of lycopene correlates significantly with the roughness of the skin," Eur. J. Pharm. Biopharm. 69(3), 943-947 (2008).

22. M. E. Darvin et al., "Influence of sun exposure on the cutaneous collagen/elastin fibers and carotenoids: negative effects can be reduced by application of sunscreen," J. Biophotonics 7(9), 735-743 (2014).

23. M. C. Meinke et al., "Bioavailability of natural carotenoids in human skin compared to blood," Eur. J. Pharm. Biopharm. 76(2), 269-274 (2010).

24. M. E. Darvin et al., "Formation of free radicals in human skin during irradiation with infrared light," J. Invest. Dermatol. 130(2), 629-631 (2010).

25. S. Vandersee et al., "Blue-violet light irradiation dose dependently decreases carotenoids in human skin, which indicates the generation of free radicals," Oxid. Med. Cell. Longevity 2015, 579675 (2015).

26. M. E. Darvin et al., "Optical methods for noninvasive determination of carotenoids in human and animal skin," J. Biomed. Opt. 18(6), 061230 (2013).

27. M. E. Darvin et al., "Comparison of two methods for noninvasive determination of carotenoids in human and animal skin: Raman spectroscopy versus reflection spectroscopy," J. Biophotonics 5(7), 550-558 (2012).

28. R. X. Yu et al., "Spectroscopic biofeedback on cutaneous carotenoids as part of a prevention program could be effective to raise health awareness in adolescents," J. Biophotonics 7(11-12), 926-937 (2014).

29. H. Lademann et al., "Non-invasive spectroscopic determination of the antioxidative status of gravidae and neonates," Skin Pharmacol. Physiol. 28(4), 189-195 (2015).

30. T. B. Fitzpatrick, "The validity and practicality of sun-reactive skin types I through VI," Arch. Dermatol. 124(6), 869-871 (1988).

31. S. Andree et al., "Evaluation of a novel noncontact spectrally and spatially resolved reflectance setup with continuously variable sourcedetector separation using silicone phantoms," J. Biomed. Opt. 15(6), 067009 (2010).

32. D. Arifler et al., "Spatially resolved reflectance spectroscopy for diagnosis of cervical precancer: Monte Carlo modeling and comparison to clinical measurements," J. Biomed. Opt. 11(6), 064027 (2006).

33. R. M. P. Doornbos et al., "The determination of in vivo human tissue optical properties and absolute chromophore concentrations using spatially resolved steady-state diffuse reflectance spectroscopy," Phys. Med. Biol. 44(4), 967-981 (1999).

34. S. A. Lisenko and M. M. Kugeiko, "Noninvasive diagnostics of skin microphysical parameters based on spatially resolved diffuse reflectance spectroscopy," J. Appl. Spectrosc. 79(6), 934-943 (2013).

35. S. Andree, C. Reble, and J. Helfmann, "Spectral in vivo signature of carotenoids in visible light diffuse reflectance from skin in comparison to ex vivo absorption spectra," Photonics Lasers Med. 2(4), 3 (2013).

36. M. Darvin et al., "Multiple spatially resolved reflection spectroscopy for in vivo determination of carotenoids in human skin and blood," Laser Phys. Lett. 13, 095601 (2016).

37. A. C. Lauer et al., "Dose-dependent vitamin C uptake and radical scavenging activity in human skin measured with in vivo electron paramagnetic resonance spectroscopy," Skin Pharmacol. Physiol. 26(3), 147-154 (2013).

38. M. C. Meinke et al., "Influence on the carotenoid levels of skin arising from age, gender, body mass index in smoking/non/smoking individuals," Free Radicals Antioxid. 1(2), 15-20 (2011).

39. S. Scarmo et al., "Significant correlations of dermal total carotenoids and dermal lycopene with their respective plasma levels in healthy adults," Arch. Biochem. Biophys. 504(1), 34-39 (2010).

40. W. Stahl et al., "Increased dermal carotenoid levels assessed by noninvasive reflection spectrophotometry correlate with serum levels in women ingesting Betatene," J. Nutr. 128(5), 903-907 (1998).

41. L. Jahns et al., "Skin and plasma carotenoid response to a provided intervention diet high in vegetables and fruit: uptake and depletion kinetics," Am. J. Clin. Nutr. 100(3), 930-937 (2014).

42. L. M. Nguyen et al., "Evaluating the relationship between plasma and skin carotenoids and reported dietary intake in elementary school children to assess fruit and vegetable intake," Arch. Biochem. Biophys. 572, 73-80 (2015).

43. P. B. Milburn, C. S. Sian, and D. N. Silvers, "The color of the skin of the palms and soles as a possible clue to the pathogenesis of acral-lentiginous melanoma," Am. J. Dermatopathol. 4(5), 429-434 (1982).

44. S. Nouveau-Richard et al., "In vivo epidermal thickness measurement: ultrasound vs. confocal imaging," Skin Res. Technol. 10(2), 136-140 (2004).

45. V. Coetzee and D. I. Perrett, "Effect of beta-carotene supplementation on African skin," J. Biomed. Opt. 19(2), 025004 (2014).

46. S. Jung et al., "Antioxidants in Asian-Korean and caucasian skin: the influence of nutrition and stress," Skin Pharmacol. Physiol. 27(6), 293-302 (2014).

47. M. C. Meinke et al., "Influence of dietary carotenoids on radical scavenging capacity of the skin and skin lipids," Eur. J. Pharm. Biopharm. 84(2), 365-373 (2013).

Martina C. Meinke studied chemistry at the Freie Universität Berlin, Germany. After graduation, she headed an environmental laboratory until she switched to medical diagnostics. Since 1999, she has been employed by the CCP. After a postgraduate study, she was awarded the title Medical Physicist in 2006. Since 2007, she has been an assistant professor of medical physics with the main focus on spectroscopy of blood and skin and electron paramagnetic resonance measurements in skin.

Silke B. Lohan studied biology at the Freie Universität Berlin. In 2013, she completed her dissertation in human genetics. In October 2013, she joined the team of the Center of Experimental and Applied Cutaneous Physiology. Her interest is focused on electron paramagnetic resonance spectroscopy of the skin, especially penetration of nanoparticles and drug release processes in the skin, and on the influence of the human antioxidant system by external triggers and its biological response.

Ihar Shchatsinin received his MS degree in physics from Belarusian State University, Minsk, in 2002. He then moved to Berlin, where he received his $\mathrm{PhD}$ in physics from the Free University in 2009 . He is now a researcher at the Laser- und Medizin-Technologie $\mathrm{GmbH}$, Berlin. His current research interests are focused on spectroscopy of biological tissues, chemometrics, and mathematical modeling.

Maxim E. Darvin studied at the Moscow State Engineering Physics Institute, the Faculty of Experimental and Theoretical Physics, Department of Medical Physics, where he completed his master's degree in physics. After a postgraduate study, he was awarded the title Dr. rer. med. from Humboldt University of Berlin, Germany, in 2007 and his PhD from Saratov State University, Russia, in 2010. His main interests are laser techniques, spectroscopy, biophysics, and skin physiology.

Björn Magnussen, after studying electrical engineering and computer science, received his doctorate degree with honors in robotics from the University of Karlsruhe. He worked extensively on automation and optical sensing methods for Siemens in Munich, Tokyo and Berkeley. After winning the Siemens "Business-Plan-Competition," he founded a technology company for miniature piezoelectric motors. From 2007, he managed the communications technology division of 
SMA solar technology. Since 2014 , he is responsible for the biozoom product development.

Wolfgang Köcher received his master's degree in engineering and his $\mathrm{PhD}$ from the University of Kassel. He was a chief engineer for systems design at the University and leader of development projects for domestic combined heat and power based on Sterling engines, Sterling air-condition systems, and systems applying $\mathrm{CO}_{2}$ as a refrigerant. After changing his scientific work to optical applications, he found the company Opsolution applying reflection spectroscopy for the development of small handheld systems.

Jürgen Helfmann, in his position as a research director of LMTB, is responsible for the medical technology resort. He also heads the resort spectroscopic methods for diagnosis and sensors in medical technology. He joined the scientific staff at the LMTB when he finished his physics degree in 1986, working alternately for the Institute of Medicine/Technical Physics and Laser Medicine at the Charité and the Laser Medizin Zentrum, now the LMTB.

Jürgen Lademann studied at the quantum electronics in the Department of the Physics, Moscow State University, Russia, where he completed his master's degree. In the year 2000, he was appointed as a professor of dermatology at the Charité-Universitätsmedizin Berlin, Germany. He is the editor of the international journal Skin Pharmacology and Applied Skin Physiology and board member of the German Federal Institute of Risk Assessment, Berlin, Germany.

Biographies for the other authors are not available. 